\title{
Surface-acoustic-wave counterflow micropumps for on-chip liquid motion control in two-dimensional microchannel arrays $\dagger$
}

\author{
Luca Masini, ${ }^{a}$ Marco Cecchini, ${ }^{* a b}$ Salvatore Girardo, ${ }^{c}$ Roberto Cingolani, ${ }^{c}$ Dario Pisignano ${ }^{c d}$ \\ and Fabio Beltram ${ }^{a b}$
}

\author{
Received 8th January 2010, Accepted 12th May 2010 \\ DOI: 10.1039/c000490a
}

Fully controlled liquid injection and flow in hydrophobic polydimethylsiloxane (PDMS) twodimensional microchannel arrays based on on-chip integrated, low-voltage-driven micropumps are demonstrated. Our architecture exploits the surface-acoustic-wave (SAW) induced counterflow mechanism and the effect of nebulization anisotropies at crossing areas owing to lateral propagating SAWs. We show that by selectively exciting single or multiple SAWs, fluids can be drawn from their reservoirs and moved towards selected positions of a microchannel grid. Splitting of the main liquid flow is also demonstrated by exploiting multiple SAW beams. As a demonstrator, we show simultaneous filling of two orthogonal microchannels. The present results show that SAW micropumps are good candidates for truly integrated on-chip fluidic networks allowing liquid control in arbitrarily shaped two-dimensional microchannel arrays.

\section{Introduction}

One of the most limiting issues for the widespread use of lab-ona-chip devices is the lack of compactness and portability of the diagnostic platforms. Indeed, the bare fluidic chip, which by itself is very compact, must be complemented with external control units, in most cases including pressurized lines as pumps for controlled liquid loading coupled with sample and reagent mlsized reservoirs. Ideally, these pumps should be miniaturized to allow integration and eliminate the need for additional specialized or bulky equipment.

Based on surface acoustic waves (SAWs), we recently introduced a novel class of fully integrated devices, demonstrating fast and versatile liquid injection in hydrophobic, PDMS-based single microchannels. ${ }^{1,2}$ It is known that when a traveling SAW interacts with a liquid droplet placed on a free surface, momentum and energy transfer from the wave to the liquid lead to a liquid response that depends on the SAW power. ${ }^{3,4}$ For high-power excitation, jet propulsion or atomization can occur. ${ }^{5}$ At low excitation power, efficient mixing is easily achieved, ${ }^{6}$ whereas at intermediate excitation power, the entire droplet translates along the SAW-propagation direction. ${ }^{7}$ In the case of liquid transfer into micro-conduits, SAWs can prove ineffective if used in this geometrical configuration (i.e. with the SAW travelling along the desired liquid motion direction). ${ }^{1}$ On the contrary, we demonstrated that SAW-induced atomization within microchannels,

${ }^{a} N E S T$, Istituto Nanoscienze-CNR and Scuola Normale Superiore, Piazza San Silvestro 12, I-56127 Pisa, Italy.E-mail: m.cecchini@sns.it; Fax: +39 050509 417; Tel: +39050 509459

${ }^{b} I I T @ N E S T$, Center for Nanotechnology Innovation, Piazza San Silvestro 12, I-56127 Pisa, Italy

${ }^{c}$ National Nanotechnology Laboratory of Istituto Nanoscienze-CNR and IIT Research Unit, Università del Salento, via Arnesano, 73100 Lecce, Italy; Fax: +39 0832298146; Tel: +390832298146

${ }^{d}$ Università del Salento, via Arnesano, 73100 Lecce, Italy

$\dagger$ Electronic supplementary information (ESI) available: Experimental set-up description, filling movies and cross-talk quantification data. See DOI: $10.1039 / \mathrm{c} 000490$ a followed by SAW-assisted coalescence, leads to very efficient liquid counterflow with respect to the SAW propagation direction [inverted drive (ID)].

By exploiting nebulization anisotropy at crossing areas induced by laterally propagating SAWs, here we introduce basic fluidic unit operations that enable liquid loading and handling in $2 \mathrm{D}$ arrays of hydrophobic microchannels. We demonstrate that, upon selectively activating single or multiple interdigital transducers (IDTs), fluids can be drawn from reservoirs (i.e. droplets at the channel entrances) into the chip micro-pipes and moved towards the desired positions of the channel grid. Motion direction and velocity are determined by the active IDT and by the SAW power, respectively. Splitting of the liquid flow can also be easily achieved by exploiting multiple SAWs propagating along different paths toward the selected split point. In this case, simultaneous filling of orthogonal microchannels is achieved.

\section{Materials and methods}

Devices are composed of two functional blocks: a fluidic layer (FL) and a SAW layer (SL). The top FL is a polydimethylsiloxane (PDMS) element with a textured microchannel array realized by replica molding technique. The geometry of the fluidic network is a $5 \times 5$ array of crossing orthogonal microchannels [Fig. 1(a)]. This architecture defines $107.5 \mathrm{~mm}-$ long microchannels and 25 squared crossing areas available for fluid loading, handling and mixing, for a total of 65-70 $\mathrm{mm}$ available fluidic path depending on the channel width. Several geometries of the channel section are examined. Specifically, channel widths span from 200 to $300 \mu \mathrm{m}$, whereas channel heights are chosen to keep constant the capillary aspect ratio (width/height $\approx 10: 1$ ). Thus, the fluidic volume of the entire chip is in the 0.65 to $0.75 \mu \mathrm{l}$ range.

The master structures for replica molding are fabricated by standard photolithography using negative SU8 photoresist deposited on silicon wafers by spin coating (see ref. 2 for details). 


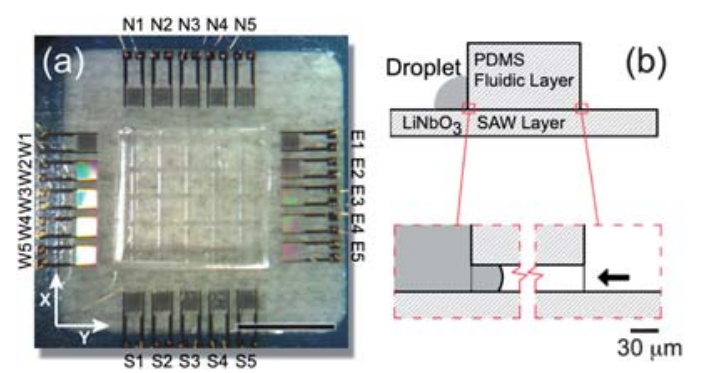

Fig. 1 (a) Photograph of a complete microfluidic chip. The PDMS fluidic layer is aligned to the metallic IDTs. Each IDT is individually labeled by a letter followed by a number: $\mathrm{N} 1-5$ and $\mathrm{S} 1-5$ are oriented along the $X$ crystallographic orientation of the $\mathrm{LiNbO}_{3}$ substrate, E1-5 and $\mathrm{W} 1-5$ along the $Y$ axis. The scale bar corresponds to $5 \mathrm{~mm}$. (b) Scheme of the device geometrical configuration. Top panel: a deionized water droplet is deposited at the microchannel entrance to serve as a liquid reservoir. Bottom panel: the black arrow indicates to the SAW propagation direction during filling.

PDMS (Sylgard 184 by Dow Corning) is then cast onto the master molds and thermal polymerization is carried out in situ at $120{ }^{\circ} \mathrm{C}$ for $60 \mathrm{~min}$. Gentle peeling off of the PDMS from the molds completes the fabrication procedure of the fluidic layer.

The bottom SL is a $\mathrm{LiNbO}_{3}$ piezoelectric substrate, with 20 IDTs for SAW excitation and detection [Fig. 1(a)]. IDTs are fabricated by standard photolithography followed by thermal metal deposition and lift-off techniques. They consist of 25 pairs of $100 \mathrm{~nm}$-thick, $800 \mu \mathrm{m}$ long Al fingers, and by Cr/Au $(25 \mathrm{~nm} /$ $260 \mathrm{~nm}$ ) bonding pads for external interfacing. The IDT periodicity is $40 \mu \mathrm{m}$ and the distance between opposed IDTs forming individual delay lines is $1 \mathrm{~cm}$. As shown in Fig. 1(a), each IDT is individually labeled with a letter and number indicating chip side and position, respectively.

Complete devices are assembled by contact superposition of the FL onto the SL (no covalent bonding was required). As shown in Fig. 1(a) and (b), PDMS microchannels are orthogonally aligned to the IDT fingers and opened to air only at the inlet/outlet positions. In this configuration, each IDT controls a specific motion direction along its corresponding microchannel.

Further descriptions of the experimental set-up for SAW excitation/detection and video acquisition/analysis are available online as ESI $\uparrow$.

The following data refer to one representative device.

\section{Results and discussion}

SAW excitation and detection are preliminarily demonstrated by measuring the scattering parameters S11, S22 and S12(S21) for each delay line. No significant difference is found among parallel delay-lines. The individual resonance frequency is dependent on the IDT orientation with respect to the substrate crystal axis. For

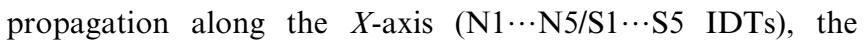
central resonance frequency is $f_{\mathrm{NS}}=97 \mathrm{MHz}$ [full width at half maximum (FWHM) of $\Delta f_{\mathrm{NS}}=2.5 \mathrm{MHz}$. Owing to the smaller

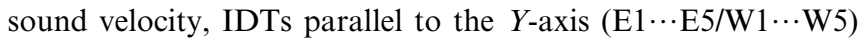
exhibit a lower resonance frequency $\left(f_{\mathrm{EW}}=91 \mathrm{MHz}\right.$, with FWHM of $\Delta f_{\mathrm{EW}}=2.5 \mathrm{MHz}$ ). Delay-line transmission characteristics [S12(S21)] demonstrate good SAW propagation (Fig. S1 $\uparrow$ ), resulting in a maximum transmittance of about -18 $\mathrm{dB}$ and $-20 \mathrm{~dB}$ for the $X$ - and $Y$-axis, respectively. The presence of the FL on top of the piezoelectric layer has no impact on optimal chip operation.

Micropumping efficiency is first tested for the case of single microchannel filling. In contrast to previous experiments, ${ }^{1,2}$ the present fluidic layout consists of discontinuous microchannels with crossing areas where the lateral constriction to the fluid motion is absent. Here only two boundaries are present (top and bottom walls) and in these conditions acoustic counter-propagation was never tested before. In this framework, Renaudin et al. ${ }^{8}$ investigated the dynamics of droplets squeezed between two planes in the presence of SAWs. In this case, however, no microchannels are present in the proximity of the fluid and authors investigated only standard droplet motion along the SAW direction.

In our experiments, $0.5 \mu \mathrm{l}$ of deionized water are loaded at the channel inlet and continuous SAWs propagating from the outlet to the inlet are excited (i.e. the ID was activated). For the present device, the power threshold activating the fluid flow is $20.6 \mathrm{dBm}$. As shown in Fig. S2, $\uparrow$ the filling velocity depends on the distance between the liquid-air interface and the active IDT and can be controlled by changing the power of the signal applied to the IDT $\left(P_{\mathrm{RF}}\right)$. SAW-action is sufficient to ensure fluid flow through the crossings (Fig. S3 $\dagger$ ). There the resulting meniscus motion slows down while remaining continuous. For example, a $(60 \pm 6) \%$ reduction of the pumping rate is measured for the $X$-direction (in this case the crossing area is located $6.6 \mathrm{~mm}$ far from the exciting IDT and $P_{\mathrm{RF}}=26.4 \mathrm{dBm}$ ). For our crossing geometry we also observe some liquid leakage into the lateral microchannels estimated as about $10 \%$ of the total injected volume (Fig. S3, $\dagger$ frame $\mathrm{t}_{4}$ ). If desired, different crossing designs can be developed to tune or avoid this effect (Fig. S4†).

To check that cross-talk between different delay lines is not relevant, we initially measure the S12(S21) parameters between IDTs lying along different delay lines. As shown in Fig. S5(a), $\dagger$ electric cross-talk is negligible. In particular, the signal detected by nearby IDTs (N1, N2, N4, and N5) is $\sim 0.01 \%$ of the signal detected by frontal IDT (N3). In order to address also any possible fluidic cross-coupling, we dispense liquid droplets at the inlet of adjacent microchannels and monitor the fluid dynamics in the presence of a single SAW beam. As shown in Fig. S5(b), $\dagger$ while the channel aligned to the active IDT is successfully filled, the liquid-air interface within the nearby microchannel remains unchanged, even at the highest $P_{\mathrm{RF}}$, thus demonstrating the independent addressability of adjacent fluidic paths. In case of extremely dense microchannel networks specifically designed IDTs like converging IDTs ${ }^{9}$ may be used to avoid possible SAW diffraction effects.

The liquid-air interface evolution at the crossings determines the direction along which the fluid motion occurs. The first fluidic unit operation enabled by counterflow is introduced in Fig. 2 $\left(t_{0}-t_{3}\right)$. It shows a typical filling sequence demonstrating flow deviation (FD), i.e. a $90^{\circ}$ directional change (see also Movie $\mathrm{S} 1 \dagger$ ). SAWs are excited by $\mathrm{N} 3\left(P_{\mathrm{RF}}=26.5 \mathrm{dBm}\right)$ and induce straight fluid propagation from the droplet reservoir into the microfluidic device. As the meniscus approaches the selected cross-region, N3 is switched off causing the liquid motion to stop [Fig. $2\left(\mathrm{t}_{0}\right)$ ]. 

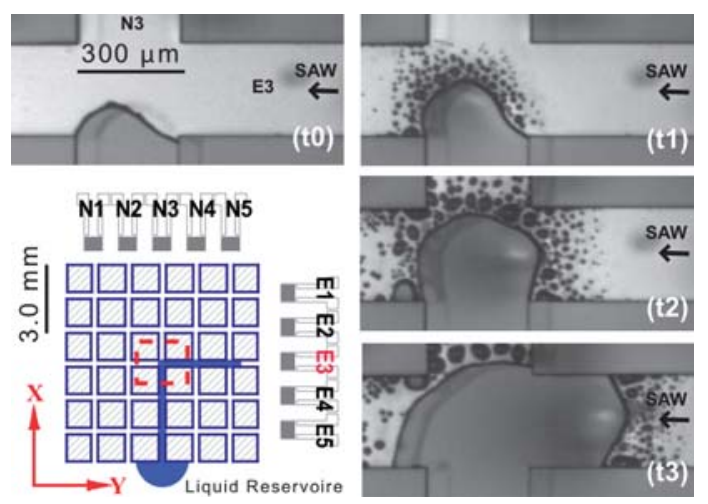

Fig. $2\left(a, t_{0}-t_{3}\right)$ Flow direction change by lateral SAW-action: typical filling sequence showing $90^{\circ}$ directional change. $\mathrm{N} 3$ is switched off when the meniscus approaches the selected cross-region causing the liquid motion to freeze $\left(\mathrm{a}, \mathrm{t}_{0}\right)$. E3 is then powered at $P=32.5 \mathrm{dBm}$. SAWs approaching from $\mathrm{E} 3$ induce enhanced nebulization at the right meniscus side $\left(a, t_{1}-t_{3}\right)$.

Orthogonal E3 is then powered $\left(P_{\mathrm{RF}}=32.5 \mathrm{dBm}\right)$. We observe a progressive filling of the cross-area and significant nebulization anisotropy at the liquid-air interface. Indeed, SAWs approaching from E3 induce enhanced nebulization at the right meniscus side. This asymmetry results in a lateral pulling force that makes the flow turn in the direction of the approaching SAW. On the contrary, nebulization at other boundaries progressively reduces until it becomes negligible owing to SAW damping at the meniscus side moving apart from the other liquid-air interfaces. Thus, the motion along $X$ and $Y$ (i.e. towards N3 and W3) stops because of the lack of nebulization, Fig. $2\left(\mathrm{t}_{3}\right)$. The pumping rate during FD can be varied by changing the lateral acoustic power. Fig. 3(a) reports the position of the liquid-air interface during lateral SAW pumping at different $P_{\mathrm{RF}}$, highlighting tunable liquid transfer by changing SAW intensity.

SAWs can also be employed to extract fluid from filled microchannels (Movie S2 $\dagger$ ). To test this second fluidic unit operation, that we name fluid extraction (FE), SAW-actuation is first used to charge an empty microchannel. The active IDT is then switched off, and orthogonal micropumping is initiated. In the specific case shown in the right panels of Fig. 3(b), SAWs are excited from E3, and the fluid behavior is monitored for $P_{\mathrm{RF}}=$ $29.4 \mathrm{dBm}$. These panels show a filling sequence where the SAW successfully pumps the fluid orthogonally to the previous motion direction. The power threshold for inducing pumping is not significantly different from that required for pumping from external droplets and indicates that no further hydraulic resistance is introduced by the division of the main fluid line. Notably, the previously charged conduit was not drained by the lateral SAW activity. The full dynamic quantification of FE is finally reported as a function of $P_{\mathrm{RF}}$ in Fig. 3(b).

The last enabling unit operation is the flow splitting (FS) at the crossings. FS can be easily obtained by exploiting multiple-SAW propagation along different paths towards selected splitting points (Movie S3†). The right panels of Fig. 3(c) illustrate this chip capability. Fluid dynamics is monitored in the presence of two converging SAW beams, specifically from N3 and E3. Thus, a single splitting point is univocally located at the cross-area between the N3-S3 and E3-W3 directions. $1 \mu 1$ of water is

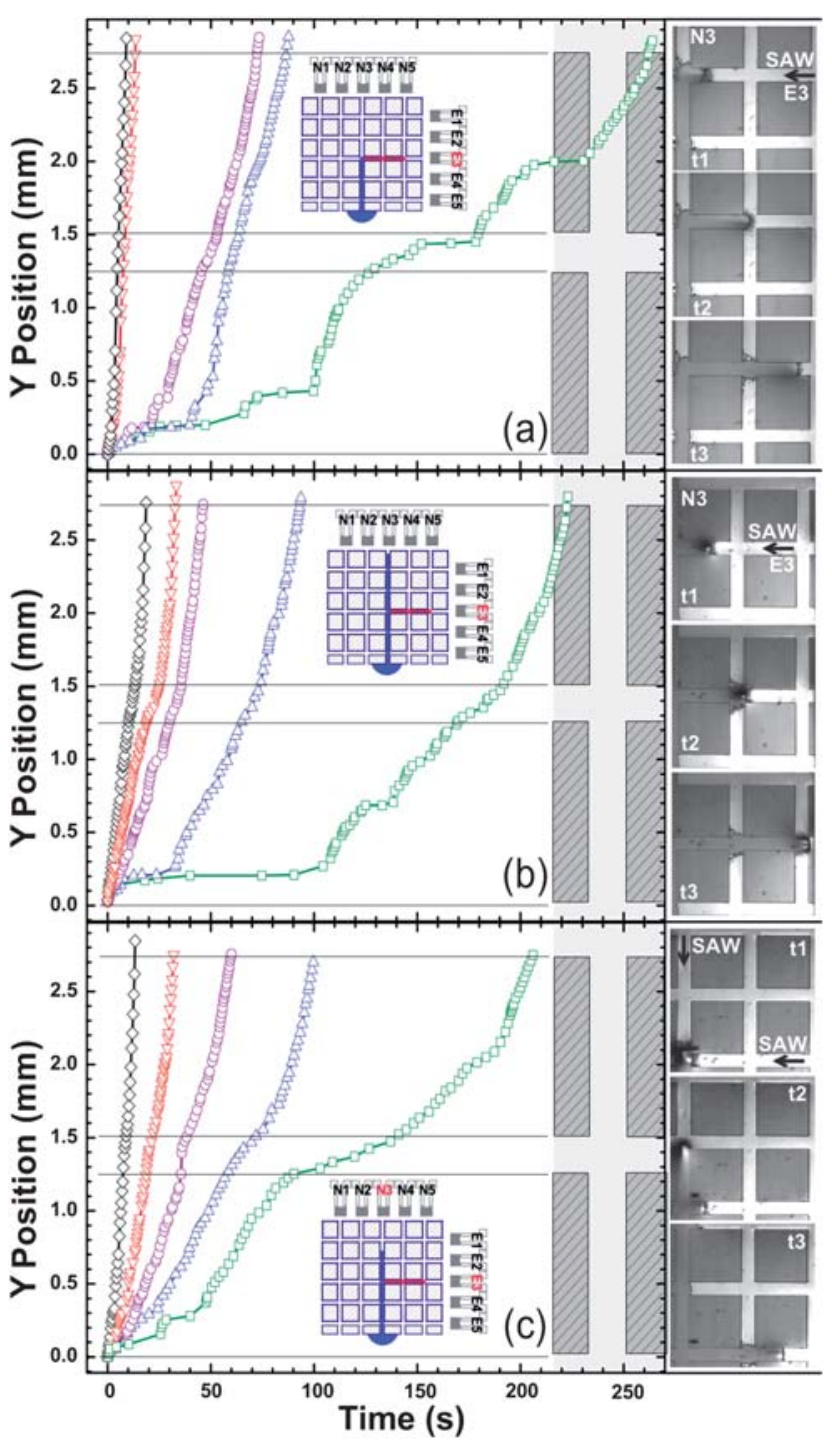

Fig. 3 Fluid dynamics by lateral SAW action: deviation (a), extraction (b) and splitting (c). Meniscus position reported as function of time for $P_{\mathrm{RF}}=28.4 \mathrm{dBm}$ (green), $29.4 \mathrm{dBm}$ (blue), $30.4 \mathrm{dBm}$ (purple), $31.4 \mathrm{dBm}$ (red) and $32.4 \mathrm{dBm}$ (black). The lateral active IDT is E3. N3 is used to address the main fluidic direction. The insets depict the fluidic configurations: red and blue lines represent lateral flows (quantified in the graphs) and the main flow direction, respectively. The right panels report selected frames from the time sequences quantified in the main graphs for the three basic unit operations.

deposited at the entrance of the N3-S3 channel, and N3 and E3 powered at the specific SAW-resonance frequencies with 28.4 and $33 \mathrm{dBm}$ respectively. The SAW beam from N3 initiates liquid motion into the chip, and single channel filling occurs until reaching the splitting point. Here, the lateral SAWs from N3 start to interact with the fluid meniscus. Enhanced and anisotropic nebulization lead to the modification of the liquid-air interface profile during the transit along the cross. As shown in the right panels of Fig. 3(c), two simultaneous pulling actions result in the main flow division and contemporary filling of orthogonally oriented microchannels. Importantly, the liquid flow can be individually tuned by varying the intensity of the 
corresponding SAW beam. As shown in the left panel of Fig. 3(c), the range of filling velocities available (of the order of $10-200 \mu \mathrm{m} \mathrm{s}^{-1}$ ) does not significantly differ from what was obtained with single IDT operation during FD and FE. In addition, we point out that the geometry of typical microfluidic devices normally does not necessitate fluidic paths greater of a few mm, a length-scale fully accessible by our micropumping approach.

The geometrical configuration of our microfluidic chip and the combination of the introduced fluidic unit operations enable very versatile liquid handling and readily allows implementing complex filling sequences. In order to show this potential, we report in Fig. S6† a possible micropumping cycle which exploits 7 different SAW-based micropumps acting along a network of 7 microchannels, containing 6 cross-areas for a total fluidic volume of $400 \mathrm{nl}$. The final liquid pattern [Fig. $\mathrm{S} 6, \uparrow$ frame $\mathrm{t}_{8}$ ] is achieved by inducing 6 direction changes followed by a fluid split.

\section{Conclusions}

In conclusion, SAW-based counterflows were successfully exploited to control liquids in hydrophobic microchannel arrays by the combination of three basic fluidic unit operations: fluid extraction, flow deviation and splitting. The devices were formed by a $5 \times 5$ orthogonal array of hybrid $\mathrm{LiNbO}_{3} / \mathrm{PDMS}$ microchannels (20 input/output ports, 25 crossing areas) and 20 IDTs for SAW excitation and detection. SAW-induced acoustic counterflow $^{1}$ was demonstrated to be capable of: (i) filling discontinuous microchannels; (ii) inducing $90^{\circ}$ flow direction changes; (iii) extracting fluid laterally from filled microchannels; and (iv) flow splitting and simultaneous multichannel filling. Finally, one example of a complex filling sequence was given showing 6 direction changes and one fluid split. In order to enable fully automated filling sequences in portable architectures, work is in progress to reduce device power consumptions and to improve the pumping-speed accuracy. There is indeed no major obstacle for the implementation of miniaturized electronics allowing the control of SAW-driven fluidic chips. As an example, we cite the recent publication by Yeo and Friend ${ }^{3}$ where Fig. 1(c) shows a photograph of a very compact power supply used by the authors to generate the SAW. In this specific case, the power supply was not much grater than a coin. In view of these results, SAW micropumps appear fully capable of serving as an enabling technology for liquid control in arbitrary shaped twodimensional fluidic networks. Thanks to the optical transparency of elastomeric materials and lithium niobate, the proposed fluidic chips are compatible with direct and inverted microscopy techniques. As an example, optical detection may be employed for biochemical essay [e.g. enzyme-linked immunosorbent assays (ELISA) or DNA microarrays]. Fluorescence imaging of biomolecules in living cells, tissues or small organisms may also represent an intriguing follow up. In this case, other than for sample loading and actuation, SAWs may also be used for cell sorting, ${ }^{10}$ concentration $^{11}$ and for applying shear stress to selected biosample regions. ${ }^{12} \mathrm{We}$ ourselves indeed successfully tested the compatibility of acoustic counterflow with fluorescent proteins $^{2}$ and, recently, other authors demonstrated that cells in the presence of SAWs offer the same functionality as untreated cells. $^{13}$

\section{Acknowledgements}

The authors are grateful for the support of the Italian Ministry of University and Research through the FIRB projects RBIN045NMB and RBLA03ER38. We also thank the Apulia Strategic Regional Project PS_144.

\section{Notes and references}

1 M. Cecchini, S. Girardo, D. Pisignano, R. Cingolani and F. Beltram, Appl. Phys. Lett., 2008, 92, 104103.

2 S. Girardo, M. Cecchini, F. Beltram, R. Cingolani and D. Pisignano, Lab Chip, 2008, 8, 1557-1563.

3 L. Y. Yeo and J. R. Friend, Biomicrofluidics, 2009, 3, 012002.

4 M. K. Tan, L. Y. Yeo and J. R. Friend, Europhys. Lett., 2009, 87, 47003.

5 M. K. Tan, J. R. Friend and L. Y. Yeo, Phys. Rev. Lett., 2009, 103, 024501 .

6 K. Sritharan, C. J. Strobl, M. F. Schneider, A. Wixforth and Z. Guttenberg, Appl. Phys. Lett., 2006, 88, 054102.

7 A. Renaudin, P. Tabourier, V. Zhang, J. C. Camart and C. Druon, Sens. Actuators, B, 2006, 113, 389-397.

8 A. Renaudin, J. P. Sozanski, B. Verbeke, V. Zhang, P. Tabourier and C. Druon, Sens. Actuators, B, 2009, 138, 374-382.

9 R. Shilton, M. K. Tan, L. Y. Yeo and J. R. Friend, J. Appl. Phys., 2008, 104, 014910.

10 T. Franke, A. R. Abate, D. A. Weitz and A. Wixforth, Lab Chip, 2009, 9, 2625-2627.

11 M. K. Tan, J. R. Friend and L. Y. Yeo, Lab Chip, 2007, 7, 618-625.

12 S. W. Schneider, S. Nuschele, A. Wixforth, C. Gorzelanny, A. Alexander-Katz, R. R. Netz and M. F. Schneider, Proc. Natl. Acad. Sci. U. S. A., 2007, 104, 7899-7903.

13 H. Li, J. Friend, L. Yeo, A. Dasvarma and K. Traianedes, Biomicrofluidics, 2009, 3, 034102-034111. 